\title{
Barriers to receiving testosterone replacement therapy in the community during COVID-19 pandemic
}

\author{
Victoria L. Troesch^, Denise Juhr, Amy Pearlman^ \\ Department of Urology, University of Iowa Hospitals and Clinics, Iowa City, IA, USA \\ Contributions: (I) Conception and design: All authors; (II) Administrative support: All authors; (III) Provision of study materials or patients: All \\ authors; (IV) Collection and assembly of data: VL Troesch, A Pearlman; (V) Data analysis and interpretation: VL Troesch, A Pearlman; (VI) \\ Manuscript writing: All authors; (VII) Final approval of manuscript: All authors. \\ Correspondence to: Victoria L. Troesch. Department of Urology, University of Iowa, 200 Hawkins Drive, 3RCP, Iowa City, IA 52242-1089, USA. \\ Email: Victoria-troesch@uiowa.edu.
}

Background: While the etiology for the upsurge in testosterone testing and prescriptions is likely multifactorial, increased direct-to-consumer marketing and the expansion of clinical care centers devoted to testosterone treatment likely play a role. Many of these centers require patients to report, in-person, on a regular basis for their injectable therapy and/or lab studies. The purpose of our study was to investigate barriers of care that patients receiving treatment for testosterone deficiency may be experiencing in the setting of COVID-19.

Methods: Our survey was posted on a closed Facebook support page for males currently receiving testosterone treatment and members of the group were invited to participate. The survey asked participants several questions related to how they received their injections, if they've experienced difficulties obtaining their injections due to COVID-19 restrictions, and about their interest in telemedicine services for their care.

Results: The majority of patients were able to receive their treatment despite barriers enforced by the pandemic. Of the 104 participants, almost half received their testosterone prescriptions from an outpatient clinic dedicated to testosterone replacement, while the other half received their therapy from a PCP, endocrinologist, or urologist. Only 5 patients (4.8\%) noted difficulties obtaining their injections during this pandemic, 4 of which received their prescriptions from dedicated testosterone clinics, and the other from a PCP. Nearly $90 \%$ of respondents self-administered their testosterone therapy. With regards to telemedicine, $57.8 \%$ of patients have utilized the technology in some capacity, however $74.4 \%$ said that they would prefer to use telemedicine video services with a urologist or APP with expertise in andrology over in-person services.

Conclusions: In our survey, the majority of the respondents have been able to receive their injectable testosterone therapy despite the ongoing pandemic. The majority of respondents self-administer their treatments, which may explain the lack of barriers. This study is the first of its kind to investigate the effect of a pandemic on the receipt of care for those being treated for testosterone deficiency with injectable testosterone.

Keywords: Testosterone replacement; testosterone replacement therapy (TRT); COVID; barriers

Submitted Apr 23, 2021. Accepted for publication Jun 30, 2021.

doi: $10.21037 /$ tau-21-350

View this article at: https://dx.doi.org/10.21037/tau-21-350

^ ORCID: Victoria L. Troesch, 0000-0001-9588-1354; Amy Pearlman, 0000-0001-9216-5387. 


\section{Introduction}

Hypogonadism or testosterone deficiency, characterized by low testosterone and symptoms including loss of libido, fatigue, decreased muscle mass, loss of body hair, hot flashes, and/or oligospermia, affects $10-14 \%$ of men aged 65 or older (1-3). Despite controversy surrounding the safety of testosterone replacement therapy (TRT) with regards to cardiovascular complications and prostate cancer, more recent work details various benefits of testosterone therapy, including increasing libido, mood, bone-mineral density, and lean body mass $(4,5)$.

Testosterone testing and prescriptions in the United States in the past 20 years have quadrupled $(6,7)$. While the etiology for the upsurge is multifactorial, increased direct to consumer advertising and the expansion of clinical care centers devoted to testosterone replacement likely play a role in an increased awareness and interest about low testosterone in the general public $(1,8)$. Standardized internet search data from Google Trend show a near tripling of search terms from the public since 2004. Urologists and endocrinologists supply only a minority of information regarding testosterone therapies on the Internet and represent only $17 \%$ of clinics most frequently encountered on standard online Google searches. The majority of information on this topic (83\%) is from dedicated testosterone therapy clinics run by nonphysicians or non-specialist providers (8). At many of these testosterone centers, patients are required to report, inperson, on a regular basis for their testosterone therapy and/ or laboratory studies. Though some of these centers may allow for patients to self-inject their testosterone at home, others necessitate in-person visits for therapy. Given the recent COVID-19 pandemic and the accompanied requisite social isolation, we suspect many patients may be at odds as they try to obtain their treatments, especially those that use clinical care center services. We aim to investigate how the current pandemic has influenced the care of patients who receive testosterone replacement therapies and barriers faced.

Additionally, for the past decade, telemedicine has been touted as one of the fastest growing aspects of the healthcare system (9). The COVID-19 pandemic has drastically affected healthcare organizations globally resulting in quick adoption and implementation of telemedicine as government regulations relaxed and reimbursement barriers were mitigated (10). Many of these changes will likely perpetuate beyond the pandemic, and we aimed to elicit the interest for the role for telemedicine with licensed urologists and advanced practice providers specifically as an alternative option for the treatment of male hormonal deficiency. We present the following article/case in accordance with the SURGE reporting checklist (available at https://dx.doi. org/10.21037/tau-21-350).

\section{Methods}

\section{Population}

Following IRB approval (exempt status) and permission from administrators of closed Facebook support groups focused on male hormonal deficiencies, an internet-based survey link was posted to chat boards and made available as part of targeted Facebook advertising.

\section{Survey design and data collection}

Two of the study investigators (VT and AP) designed a 17 question, self-administered online survey based upon previous information in the literature about testosterone administration and clinical experience. An online survey program (RedCap) was used to administer the survey. The study was conducted in accordance with the Declaration of Helsinki (as revised in 2013). The study was approved by the institutional review board at the University of Iowa (No.: IORG0000070) and individual consent for this retrospective analysis was waived.

After obtaining permission from the administrator of each website, a notice was placed explaining the study's purpose and displaying a link to the survey. Additionally, targeted ads were posted on Facebook. The survey was open for 16 weeks beginning in April 2020. Inclusion criteria included men $\geq 18$ years old currently receiving injectable testosterone therapy. Survey responses were not linked to any specific individual and no identifying patient health information was collected. Instructions included the statement, "You are free to skip any questions that you prefer not to answer." Surveys with incomplete responses were not excluded, resulting in variations in sample sizes for different variables, however analysis included only those participants that completed at least $75 \%$ of the survey. No financial incentive was offered to participants.

\section{Survey characteristics}

Participants who indicated that they were 18 years of age or older and currently on injectable testosterone therapy were directed to the survey questions. No additional questions 
were asked for those who answered "no" to either of these screening questions. Details regarding clinic and provider currently responsible for testosterone administration, as well as barriers encountered due to the COVID-19 pandemic, were assessed. Additional questions inquired about past use and interest in telemedicine services. There were no further attempts to contact participants.

\section{Statistical analysis}

Simple frequencies were calculated for respondent characteristics and responses to survey questions.

\section{Results}

Of 119 respondents, 104 met inclusion criteria. Of these, almost half $(45.2 \%)$ received their testosterone prescriptions from an outpatient clinic dedicated to testosterone replacement, $19.2 \%$ from their primary care provider, $13.5 \%$ from a urologist, and $7.7 \%$ from an endocrinologist. The remainder of respondents received their treatments from a functional medicine physician or an advanced practice provider. Ninety-two of the 104 individuals (88.5\%) self-administered injections. The remaining twelve respondents received their testosterone therapy in person at a clinic by a health care provider. If offered instruction on how to perform self-administered injections of testosterone, 5 of the 12 men $(41.7 \%)$ stated they would prefer it as an alternative to presenting for in-person testosterone injections. Only 5 of the 104 men $(4.8 \%)$ had difficulties obtaining their injection due to COVID-19 restrictions. Of these 5 men, 4 (80\%) obtained their prescriptions from outpatient clinics dedicated to testosterone therapy. Additionally, of those that endorsed difficulty obtaining their injections, two individuals sought their testosterone replacement from the black market. The majority of men $(48.0 \%)$ had an appointment in clinic either to receive injections or meet with their testosterone-prescribing practitioner every 3 months. Others met with practitioners weekly $(6.9 \%)$, monthly (11.8\%), biannually $(22.5 \%)$, or annually $(5.8 \%)$.

With regards to telemedicine, 52 of 88 respondents (59.0\%) participated in telemedicine care using two-way video in the past. If offered telemedicine video services with a urologist or an advanced practice provider with expertise in andrology for the evaluation and follow-up monitoring of their testosterone therapy, $74.4 \%$ of individuals stated they preferred this service over in-person services. Furthermore, over half of respondents indicated willingness to pay cash for telemedicine services, ranging from \$5-400 (average 88.50) for a new visit and $\$ 5-150$ (average 51.57) for a return visit. Others interested in telemedicine endorsed a willingness to pay their insurance co-pay, but denied interest in cash pay services.

\section{Discussion}

Over the last few decades, there has been a significant increase in testosterone prescriptions in the United States. Increased direct to consumer advertising and clinics dedicated to testosterone replacement therapies likely play a role. Our study is the first of its kind to investigate the effect of a pandemic on the receipt of care for those being treated for testosterone deficiency with injectable testosterone.

Our data show that the majority of respondents have been able to receive their injectable testosterone therapy despite the ongoing pandemic. In fact, less than $5 \%$ of respondents indicated any difficulty in obtaining their therapy despite the ensuing restrictions as a sequelae of the pandemic. The majority of respondents self-administer their testosterone therapy, which may explain the lack of barriers. We are unable to ascertain if testosterone administered in-person is an obstacle to treatment as a minority of respondents indicated in-person administration. However, patient education and teaching may play a role in increased access to care and ultimately lower healthcare costs with decreased number of outpatient visits. Despite our small sample size, nearly half of patients reporting for in-person injections stated they would prefer at home selfadministration if available. Clinics supplying testosterone therapies should be encouraged to teach interested patients self-injection as this may improve access to care and increase patient satisfaction.

Interestingly, almost two-thirds of the men surveyed met with their testosterone prescriber weekly, monthly, or every 3 months. We did not ask subjects why such frequent follow up was performed or how often their TRT regimens were changed to warrant this close follow up, however the frequency is significantly greater than current recommendations by the AUA. Current guidelines suggest an initial follow up testosterone level after initiation of therapy to ensure target levels have been achieved, followed by repeat measurements every 6-12 months while on therapy (11). Anecdotally, patients have reported more frequent lab draws and follow up at dedicated testosterone therapy clinics than at a urologist's office. Half of our 
respondents received their treatments at dedicated clinics, so this may contribute to the frequency of follow-up. Further investigations are necessary to fully elucidate these factors.

Another avenue shown to increase access to care is telemedicine (9). Although just over half of respondents had utilized this technology in the past, nearly $75 \%$ indicated interest in telemedicine services for their TRT with a licensed urologist or APP over in-person visits. This mirrors the results of a recent study of general urologic patients, in which $84.7 \%$ of patients stated they would prefer a telemedicine rather than a face-to-face consultation with a urologist (12). Telemedicine with licensed practitioners may provide an important adjunct to care irrespective of the status of the pandemic.

Our study was not without limitations. As surveys were sent out to testosterone therapy support groups, the pool of respondents may not accurately represent the broader population of patients treated for testosterone deficiency. There was an inherent selection bias towards individuals who have access to the Internet and choose to become a part of a support group. Our study may have been biased towards individuals that are internet knowledgeable and thus may be more likely to express interest in telemedicine services. Additionally, when inquiring about interest in telemedicine services, survey questions did not differentiate between telemedicine with specialist vs non specialists. Thus, respondents may have been indicating the desire for specialist services as opposed to telemedicine services. Although this study relied on self-reported data and its perceived limitations, Internet-based questionnaires have been shown to produce data that are equivalent to the quality of studies using traditional survey methods (13).

In conclusion, despite the novel coronavirus pandemic, men receiving testosterone therapy expressed few barriers in obtaining their treatments. Although four of the five men that endorsed barriers received their care from a clinic dedicated to testosterone treatments, our sample size was not large enough to draw any discrete conclusions. The majority of men self-administer their testosterone injections, which may account for the lack of barriers seen in our study. Patient educational tools on self-injection should continue to be encouraged to improve access to care. There is significant patient interest in telemedicine as a modality of care for their low testosterone, which may become an important tool for clinical care beyond the pandemic.

\section{Acknowledgments}

The abstract in this manuscript was presented at the 21st Annual Fall Scientific Meeting of the Sexual Medicine Society of North America in 2020. All abstracts presented at this meeting are published in the Fournal of Sexual Medicine. Funding: None.

\section{Footnote}

Reporting Checklist: The authors have completed the SURGE reporting checklist. Available at https://dx.doi. org/10.21037/tau-21-350

Data Sharing Statement: Available at https://dx.doi. org/10.21037/tau-21-350

Peer Review File: Available at https://dx.doi.org/10.21037/ tau-21-350

Conflicts of Interest: All authors have completed the ICMJE uniform disclosure form (available at https://dx.doi. org/10.21037/tau-21-350). AP is a consultant for Endo Pharmaceuticals. The other authors have no conflicts of interest to declare.

Etbical Statement: The authors are accountable for all aspects of the work in ensuring that questions related to the accuracy or integrity of any part of the work are appropriately investigated and resolved. The study was conducted in accordance with the Declaration of Helsinki (as revised in 2013). The study was approved by the institutional review board at the University of Iowa (No.: IORG0000070) and individual consent for this retrospective analysis was waived.

Open Access Statement: This is an Open Access article distributed in accordance with the Creative Commons Attribution-NonCommercial-NoDerivs 4.0 International License (CC BY-NC-ND 4.0), which permits the noncommercial replication and distribution of the article with the strict proviso that no changes or edits are made and the original work is properly cited (including links to both the formal publication through the relevant DOI and the license). See: https://creativecommons.org/licenses/by-nc-nd/4.0/.

\section{References}

1. Layton JB, Li D, Meier CR, et al. Testosterone lab 
testing and initiation in the United Kingdom and the United States, 2000 to 2011. J Clin Endocrinol Metab 2014;99:835-42.

2. Bandari J, Ayyash OM, Emery SL, et al. Marketing and Testosterone Treatment in the USA: A Systematic Review. Eur Urol Focus 2017;3:395-402.

3. Ross A, Bhasin S. Hypogonadism: Its Prevalence and Diagnosis. Urol Clin North Am 2016;43:163-76.

4. Conners WP 3rd, Morgentaler A. The evaluation and management of testosterone deficiency: the new frontier in urology and men's health. Curr Urol Rep 2013;14:557-64.

5. Traish AM. Benefits and Health Implications of Testosterone Therapy in Men With Testosterone Deficiency. Sex Med Rev 2018;6:86-105.

6. Tsametis CP, Isidori AM. Testosterone replacement therapy: For whom, when and how? Metabolism 2018;86:69-78.

7. Baillargeon J, Urban RJ, Ottenbacher KJ, et al. Trends in androgen prescribing in the United States, 2001 to 2011. JAMA Intern Med 2013;173:1465-6.

Cite this article as: Troesch VL, Juhr D, Pearlman A. Barriers to receiving testosterone replacement therapy in the community during COVID-19 pandemic. Transl Androl Urol 2021;10(8):33123316. doi: 10.21037/tau-21-350
8. Oberlin DT, Masson P, Brannigan RE. Testosterone replacement therapy and the internet: an assessment of providers' health-related web site information content. Urology 2015;85:814-8.

9. Ellimoottil C, Skolarus T, Gettman M, et al. Telemedicine in Urology: State of the Art. Urology 2016;94:10-6.

10. Contreras CM, Metzger GA, Beane JD, et al. Telemedicine: Patient-Provider Clinical Engagement During the COVID-19 Pandemic and Beyond. J Gastrointest Surg 2020;24:1692-7.

11. Mulhall JP, Trost LW, Brannigan RE, et al. Evaluation and management of testosterone deficiency: AUA guideline. $\mathrm{J}$ Urol 2018;200:423-32.

12. Boehm K, Ziewers S, Brandt MP, et al. Telemedicine Online Visits in Urology During the COVID-19 Pandemic-Potential, Risk Factors, and Patients' Perspective. Eur Urol 2020;78:16-20.

13. Gosling SD, Vazire S, Srivastava S, et al. Should we trust web-based studies? A comparative analysis of six preconceptions about internet questionnaires. Am Psychol 2004;59:93-104. 